\section{BASEM 2002 Silver Jubilee Congress}

\section{THE FIST SEPARATION SIGN: AN ADJUNCT TO SELECTION OF THE ANTERIOR CRUCIATE LIGAMENT DEFICIENT KNEE REQUIRING RECONSTRUCTION}

R.T. Roach', I. Dos Remedios, D. Griffiths, D. Rees, N. Maffulli. North Staffs Royal Infirmary, University of Keele, Stoke on Trent, Staffordshire, UK; 'Robert Jones and Agnes Hunt Hospital, Oswestry, Shropshire, UK

We designed a retrospective and prospective study to analyse the presence of perceived linear separation in the anterior cruciate ligament $(A C L)$ injured knee. This information is easily elicited by mimicking the knee joint as two fists articulating with each other.

Retrospective recall is poor before reconstruction, but the sign remains absent after successful surgery. It is also absent in any patient selecting conservative treatment and control normal knees. However, the sign is present in arthroscopically proven completely deficient $\mathrm{ACL}$ knees warranting reconstruction on the basis of pivotal instability, but is not so convincing with the partial tear or $\mathrm{ACL}$ on posterior cruciate ligament subgroups. In the prospectively followed patients, the perceived separation clearly resolves with surgery.

The fist separation sign is easy to elicit and should be included as an adjunct in the initial consultation. It can be used as a quick screening question to aid diagnosis and facilitate fast track referral. Patients can also be confidently informed that this perceived feeling will improve with reconstruction.

\section{ROLE OF EXERCISE IN MULTIPLE SCLEROSIS}

E. Curry, A.J. Maile. Dept of Physical Education, Sport and Leisure Studies, University of Edinburgh, Edinburgh, Scotland, UK

Multiple sclerosis (MS) is a chronic inflammatory disease of the central nervous system where it produces widespread demyelination' causing symptoms such as fatigue and muscle weakness, ${ }^{2}$ which can be seen to have effects on a patient's capability to exercise. This project aimed to investigate whether exercise could have a positive effect on disease symptoms as evidence for this is emerging.

Twenty patients (six men and 14 women, mean age 44.2 years), of moderate disability ${ }^{3}$ were split into two groups: exercise group (seven women, three men; mean (SD) age 42.1 (1.6) years); control group (seven women, three men; mean (SD) age 43.3 (2.3) years). Exercise testing consisted of subjects performing a five minute walk at a pace comfortable to each. Heart rate (HR) and rating of perceived exertion (RPE) were measured. An eight week training programme followed for the exercise group, with testing for all at the end. Profile of mood states (POMS) administration also occurred before and after the eight week period.

Significant improvements occurred in the exercise test performance of the exercise group $(p<0.05)$. No significant differences were found for HR and RPE scores. The exercise group also improved their POMS scores for vigour, depression, and fatigue following the eight week period.

This study has shown that exercise can be beneficial to an MS population in symptom management. Further research is required using larger samples and a greater variety of disability in subjects.

1. Herndon RM. In Diagnosis, medical management and rehabilitation. Demos, 2000:35-48.

2. LaRocca NG. In Diagnosis, medical management and rehabilitation. Demos, 2000:405-24.

3. Petajan JH, White A. Sports Medicine 1999.

\section{PERFORMANCE ENHANCING DRUGS: WHAT ARE THEY DOING?}

J.R. Locker, C.J. Bacon', W.L. Ledger. Academic Unit of Obstetrics \& Gynaecology, Jessop Wing, RHH, Sheffield S1O 2SF, UK; ' School of Sport \& Leisure Management, Sheffield Hallam University, Sheffield S1O 2BP, UK

To explore reasons for use and perceived effects of performance enhancing substances in bodybuilding, a systematic literature review was undertaken along with semistructured interviews with three users of such drugs. A Medline search using combinations of the terms "anabolic steroids", "health", "sport", and "infertility" yielded 60 relevant articles in the English language. Evidence from both the literature review and interviews suggests that use of a range of performance enhancing drugs extends beyond the world of elite sport into that of recreational bodybuilding. ' Estimates of prevalence from the three bodybuilders interviewed suggest that the degree of use may be more widespread than previously thought. They reported using anabolic steroids-for example, Sustanon, Deca-Durabolin, Testex, Winstrol, Pro-Viron, Delatestryl-ephedrine, growth hormone, insulin, insulin-like growth hormone, Clenbuterol, tamoxifen, Anastrozole, dinitrophenol (DNP), $\gamma$-hydroxybutyrate (GHB), thyroxine, HCG, caffeine, aspirin, L-Dopa, Clomiphene, and AGE (advanced glycolysation end-product) crosslink breakers. These drugs were obtained from gyms, stolen from hospitals, imported on the black market, and were often obtained more cheaply than NHS prices. The three users were extremely well informed about the substances they were taking and the effect on their bodies, yet they made informed choices to continue to use them. More research into the long term effects is needed in order for sportspeople engaging in these practices to be better advised by the medical profession.

1. Korkia P, Stimson GV. Int J Sports Med 1997

\section{O05 PREVALENCE OF ACETABULAR LABRUM TEARS IN ATHLETES PRESENTING WITH GROIN PAIN}

A.A. Narvani, E. Tsiridis, C.C. Tai, R. Chaudhuri, P. Thomas. School of Human Health and Performance, University College London, Archway Campus, Level 2 Holborn Union Building, 10 Highgate Hill, London N19 3UA, UK

Aims: To investigate the prevalence of acetabular labrum tears in athletes presenting with groin pain.

Methods: We prospectively studied the prevalence of acetabular tears in 18 athletes presenting with groin pain to our sports clinic. Clinical examination and magnetic resonance arthrography (MRA) assessed presence or absence of acetabular labrum tears. Ethical committee approval and informed consent from each patient were obtained.

Results: In four out of 18 athletes (22\%) the MRA showed the presence of an acetabular labrum tear. Clicking sensation of the hip was a sensitive $(100 \%)$ and specific $(85 \%)$ clinical symptom to predict labral tears. Internal rotation-flexion-axial compression manoeuvre was sensitive $(75 \%)$ but not specific $(43 \%)$. Thomas test was neither sensitive nor specific.'

Conclusions: Acetabular labrum tears can be a common cause of groin pain in athletes. MRA of the hip is a valuable tool in diagnosing this pathology.

1. Fitzgerald $\mathrm{RH}$. Acetabular labrum tears. Diagnosis and treatment. Clin Orthop 1995;311:60-8.

\section{CHANGES IN HAEMOSTASIS WITH EXERCISE IN ATRIAL FIBRILLATION BEFORE AND AFTER DC CARDIOVERSION}

E. Mackie, L. McKenna, T. Brummitt, M. Dawson, A. McKenzie, W.S Hillis. Department of Medicine and Therapeutics, University of Glasgow, 38 Church Street, Glasgow G12 8QQ, Scotland, UK

Aim: To assess if DC cardioversion for atrial fibrillation (AF) is beneficial in improving functional capacity and assess the haemostatic response to exercise.

Methods: Twenty patients with AF and 12 healthy controls performed a Bruce protocol exercise test. Blood sampling was performed before, after, and 30 minutes after exercise for platelet count, prothrombin time (PT), activated partial thromboplastin time (APTT), and plasma fibrinogen. Platelet activation was assessed by flow cytometry using CD62 (p-selectin) and antibodies to fibrinogen at rest and in response to ADP and adrenaline (epinephrine). The protocol was repeated one month after cardioversion.

Results: Patients with AF had higher plasma fibrinogen levels $(4.56$ (1.69) v $2.06(0.3) ; p=0.0001), \%$ CD62 (1.47 (1.5) v $0.63(0.4)$; 
$p=0.1)$, and \% fibrinogen antibody $(21.4(21.2)$ v $5.0(7.35) ; p=$ $0.05)$ than healthy controls. Peak $\mathrm{VO}_{2}$ was reduced in patients $(20.6$ (5.6) v 42.9 (4.1): $p=0.0001$ ), with no improvement seen after successful cardioversion (20.6 (4.7) v 19.6 (3.9); $p=0.88)$. Platelet activation shown by both CD62 and anti-fibrinogen was reduced after exercise (CD62: 1.47 (1.5) v 0.67 (0.39), $p=0.01$; anti-fibrinogen: $21.38(21.2) \vee 8.77(20.6), p=0.05)$. Successful cardioversion was associated with a reduction in CD62 at rest (1.47 (1.5) v 1.02 (0.7); $\mathrm{p}=0.043)$. PT and APTT shortened with exercise on both tests $(30.1$ (9.9) v $27.2(9.3), p=0.01 ; 39.8(6.0)$ v $37.8(5.9), p=0.002)$

Conclusion: Despite a resting prothrombotic state, exercise in AF appears beneficial in reducing platelet activation. The introduction of an exercise training programme in this group may have significant benefits in reducing thrombotic risk.

\section{PROMOTION OF PHYSICAL ACTIVITY THROUGH PRIMARY CARE: BELIEFS, CONCERNS, AND SELF REPORTED PRACTICE OF GENERAL PRACTITIONERS}

E.M. Board', J. Thatcher'. 'Division of Sport and Exercise Sciences, School of Social Sciences, University of Teesside, Borough Road, Middlesbrough TS1 3BA, UK; ${ }^{2}$ Sport, Health and Exercise Sciences, St Mary's College, Waldegrave Road, Strawberry Hill, Twickenham, UK

Physical activity promotion remains a relatively new public health initiative in the UK. The purpose of this study was to examine the beliefs, concerns, and self reported referral practice of general practitioners (GPs) towards the promotion of physical activity and the provision of exercise referral (ER) schemes. A questionnaire that examined personal exercise behaviour and exercise promotion, local ER scheme provision, and the perceived value, risks, concerns, and effectiveness of ER was posted to 89 GPs in the North East of England. Interviews were conducted with nine. Frequencies were conducted to describe the response data and $\chi^{2}$ analyses were performed to compare responses by GP personal exercise behaviours and exercise referral behaviours. Forty eight (54\%) GPs responded to the questionnaire, $90 \%$ of whom believed exercise is beneficial for general health and $71 \%$ engaged regularly in physically active. Some $85 \%$ of the GP surgeries offered exercise advice, and $81 \%$ of GPs currently refer patients to local ER schemes. ER schemes were generally considered innovative, valuable interventions. Only 9\% of GPs emphasised their dissatisfaction with local ER schemes, reporting a series of system (time, lack of feedback, administrative burden) and attitudinal (long term efficacy and unresolved medicolegal issues) barriers. In conclusion, GPs felt positive towards ER schemes and believed their place was complementary to, not replacement for, other interventions.

\section{THE SIDE OF SPONDYLOLYSIS: A RETROSPECTIVE COHORT STUDY OF THOSE INVESTIGATED WITH SINGLE PHOTON EMISSION COMPUTERISED TOMOGRAPHY AND REVERSE GANTRY COMPUTERISED TOMOGRAPHY}

P.L. Gregory, M.E. Batt, R.W. Kerslake, J.K. Webb. Queen's Medical Centre, Nottingham, UK

Spondylolyses are stress fractures of the pars interarticulares. Participation in sport does not give rise to symmetrical stresses on the skeletal system, and hence there may be a preponderance of spondylolyses on one side of the neural arch. The aim of this study was to determine the characteristics of those investigated for spondylolysis and to establish whether this lesion is more common on either side of the neural arch.

Medical records of 213 (131 male, 82 female) patients investigated for spondylolysis at Queen's Medical Centre, Nottingham between 1 January 1995 and 31 December 2000 were reviewed. The median age of onset of low back pain was 17.3 years (range 6.4-46.5). Most were known to have been involved in sport $(79.3 \%)$. There were 42 cricketers, 28 soccer players, 13 gymnasts, and 12 rugby footballers. Lateralisation of the low back pain occurred in $49.3 \%$, with 66 having more left sided pain and 39 more right sided pain $\left(p<0.01 ; \chi^{2}\right.$ test).

A pronounced increase in scintigraphic uptake was noted on the left side of the neural arch in 31 patients and on the right in 17 $(p<0.05)$. Spondylolysis was identified in 81 patients. Spondylolyses were bilateral on 44 occasions, but unilateral lesions occurred in the left pars 36 times and on the right 16 times $(p<0.01)$.

We conclude that spondylolyses occur more commonly in the left pars interarticulares in young English sports people. This may be

\begin{tabular}{|c|c|c|c|}
\hline \multicolumn{4}{|c|}{ Abstract 8} \\
\hline $\begin{array}{l}\text { Lumbar } \\
\text { level }\end{array}$ & $\begin{array}{l}\text { Increased } \\
\text { scintigraphic } \\
\text { activity on } \\
\text { SPECT }\end{array}$ & $\begin{array}{l}\text { Spondylolysis } \\
\text { identified on CT } \\
\text { (complete) }\end{array}$ & $\begin{array}{l}\text { Spondylolysis } \\
\text { identified on C1 } \\
\text { (incomplete) }\end{array}$ \\
\hline L1 & 12 & 0 & 1 \\
\hline L2 & 32 & 5 & 1 \\
\hline L3 & 71 & 13 & 3 \\
\hline L4 & 57 & 10 & 12 \\
\hline L5 & 126 & 77 & 21 \\
\hline Total & 145 & 105 & 38 \\
\hline
\end{tabular}

because fast bowlers tend to pars defects on the contralateral side to their bowling arm. 'This hypothesis will be duly tested on this dataset.

1. Hardcastle P, Annear P, Foster DH, et al. J Bone Joint Surg $[\mathrm{Br}]$ 1992

\section{EFFECTS OF ACUTE DYNAMIC EXERCISE ON HAEMOSTASIS AFTER MYOCARDIAL INFARCTION}

D. Carrick, L. Jamieson, M. Dawson, A. McKenzie, E. Mackie, W.S. Hillis. Department of Medicine and Therapeutics, University of Glasgow, 38 Church Street, Glasgow G12 8QQ, Scotland, UK

Background: An increased risk of acute coronary events had been documented after strenuous exercise.

Aim: To assess the thrombotic risk associated with exercise testing four weeks after myocardial infarction (MI) and assess the effects of cardiac rehabilitation with repeat testing at 12 weeks.

Methods: Thirty five Ml patients and 12 healthy controls completed a Bruce protocol exercise test with venous sampling before, after, and 30 minutes after exercise for platelet count, prothrombin time, activated partial thromboplastin time, and fibrinogen. Platele activation was assessed by flow cytometry using CD62 (p-selectin) and antibodies to fibrinogen.

Results: Exercise time improved significantly after rehabilitation (579.3 $\vee 499.1$ seconds; $p=0.003)$, although it did not match healthy controls. Platelet activation was reduced at 12 weeks compared with at four weeks, although it remained higher than controls. Healthy controls showed significant platelet activation with exercise, an effect not seen in the patient group.

Conclusion: The benefits of cardiac rehabilitation have been shown with improved exercise time and reduced platelet activation. No increased risk in terms of platelet activation were found with exercise after $\mathrm{Ml}$, and the contrasting effect in the controls suggests that platelet activation with exercise is intensity dependent.

\section{CLOPIDOGREL INHIBITS PLATELET ACTIVATION AND EXERCISE INDUCED ISCHAEMIA IN STABLE CORONARY ARTERY DISEASE}

I. Thomson, J. Sandhu, P. Langley, M. Dawson, A. McKenzie, E. Mackie W.S. Hillis. Department of Medicine and Therapeutics, University of Glasgow, 38 Church Street, Glasgow G12 8QQ, Scotland, UK

Hypothesis: Clopidogrel inhibits platelet activation and provides additional benefits over aspirin in the prevention of exercise induced haemostatic changes.

Methods: Fifteen patients with ischaemic heart disease (IHD) awaiting $\mathrm{PCl}$ performed two Bruce protocol exercise tests while receiving aspirin $75 \mathrm{mg}$ or aspirin $75 \mathrm{mg} /$ clopidogrel $75 \mathrm{mg}$. Blood sampling was performed before, after, and 30 minutes after exercise. Platelet activation was assessed by flow cytometry using CD62 (p-selectin) and antibodies to fibrinogen at rest and with agonists, ADP, and adrenaline (epinephrine)

Results: Exercise time increased while on clopidogrel $1500(112)$ seconds to 579 (129) seconds; $p=0.003)$. Exercise induced ST depression was reduced on clopidogrel $(-1.9(0.9) v-1.0(0.6) ; p=$ 0.04 ). Despite an increase in platelet count no significant changes in resting platelet activation markers were seen with exercise. Fibrinogen antibody binding was reduced by clopidogrel at rest $110.9 \% 195 \%$ confidence interval (Cl) 3.4 to 22.9$)$ v $3.6 \%(95 \% \mathrm{Cl} 1.1$ to 5.8$) ; \mathrm{p}=$ $0.08)$ as was total serum fibrinogen $(2.98(95 \% \mathrm{Cl} 2.5$ to 3.3$) \vee 2.53$ 
(95\% Cl 2.24 to 2.9); $p=0.06$ ). Clopidogrel abolished the platelet response to ADP at all stages of exercise, but most significantly immediately after exercise (\% CD62: $39.9(95 \% \mathrm{Cl} 37.2$ to 60.4$)$ v 21.6 $(95 \% \mathrm{Cl} 18.9$ to 33.9$), p=0.008$; \% anti-fibrinogen: $79.0195 \% \mathrm{Cl}$ 55.2 to 92.0$)$ v $38.1(95 \% \mathrm{Cl} 20.8$ to 53.2$), p=0.009)$ ).

Conclusion: In contrast with our data in normal subjects, stable IHD patients show no significant changes in platelet activation with exercise. Clopidogrel improves functional capacity and exercise induced ST changes, suggesting that even in stable angina, superimposed thrombus may be an important factor in precipitating ischaemia, requiring more aggressive anti-platelet treatment than aspirin alone.

\section{EXERCISE TESTING IN ADULT CONGENITAL PATIENTS WITH COARCTATION OF THE AORTA}

J. Jibu, C. Marshall A. Mckenzie, E. Mackie, W.S. Hillis. Department of Medicine and Therapeutics, 38 Church Street, Glasgow G12 8QQ, Scotland, UK

Aim: To compare exercise capacity in an aortic coarctation population with age, sex matched healthy controls and relate this to the echocardiographic and magnetic resonance imaging (MRI) findings as well as reported physical activity levels.

Methods: Thirteen patients and 13 controls performed a Bruce protocol exercise test with continuous expired gas analysis for measurement of peak $\mathrm{VO}_{2}$. Blood pressure was recorded at rest and 1 , 3, 5, and 10 minutes after exercise. Questionnaires asking for details of average weekly physical activity were completed by all patients. Variables were correlated with the MRI and echocardiographic findings.

Results: Patients had significantly elevated heart rate and systolic blood pressure $(\mathrm{p}<0.05)$. Exercise duration, peak $\mathrm{VO}_{2}, \mathrm{~V}_{\mathrm{CO}_{2}}$, and maximum heart rate were significantly lower in the patient group $(p<0.01)$. No correlation was found between exercise capacity and MRI and echocardiographic measurements. Exercise capacity did correlate with physical activity scores assessed by questionnaire.

Conclusions: Aortic coarctation results in reduced exercise capacity relative to healthy controls. However, the elevated resting heart rate and correlation with physical activity score rather than objective imaging methods suggests that this relates to level of physical fitness rather than underlying structural abnormality.

\section{FACTORS AFFECTING EXERCISE CAPACITY IN ADULTS WITH TETRALOGY OF FALLOT}

J.P. Seely, J.H.N. Fisher, D. Jenkins, E. Mackie, W.S. Hillis. Department of Medicine and Therapeutics, University of Glasgow, 38 Church Street, Glasgow G12 8QQ, Scotland, UK

Aim: Tertralogy of Fallot is the commonest cyanotic congenital cardiac lesion consisting of pulmonary oufflow tract stenosis, ventricular septal defect, overriding aorta, and right ventricular hypertrophy. Residual cardiac lesions are common, therefore we aimed to investigate the effect of these on exercise capacity as well as to correlate physical activity levels with objective measurements of exercise capacity.

Methods: Nine patients with tetralogy of Fallot and nine healthy controls performed a full Bruce protocol exercise test with continual expired gas analysis to determine peak $\mathrm{Vo}_{2}$. All subjects completed a physical activity questionnaire, and the scores were correlated with exercise results and echocardiographic findings.

Results: Significant correlations were found between peak $\mathrm{VO}_{2}$ and physical activity scores $(p=0.02)$. Regression analysis showed a significant relation between heart rate response at peak exercise and peak $\mathrm{VO}_{2}(p=0.03)$. No association was found between degree of pulmonary regurgitation and peak $\mathrm{VO}_{2}$. The presence of a residual ventricular septal defect had no effect on any variables measured.

Conclusion: The relation between peak heart rate and $\mathrm{VO}_{2}$ suggests chronotropic incompetence as a mechanism for reduced exercise capacity in this group. The lack of association between pulmonary regurgitation may relate to the degree of pulmonary regurgitation in the group studied and cannot be extrapolated beyond this. Physical activity questionnaires reflect peak $\mathrm{VO}_{2}$ achieved and could be an additional, effective tool to use in the assessment and of these patients.
015 RADIOLOGICAL SCREENING OF THE CERVICAL SPINE OF PROFESSIONAL RUGBY PLAYERS FOR CONGENITAL, DEVELOPMENTAL, AND ACQUIRED ABNORMALITIES

J.C. Peyrin, J.H. Tourette, B. Castinel.

Background: After a scrum accident resulting in tetraplegia, magnetic resonance imaging (MRI) showed spinal cord concussion caused by a voluminous herniated disk associated with cervical canal stenosis.

Aim: This accident and its consequences prompted us to reconsider the problem of detecting congenital, developmental, and acquired abnormalities, especially canal stenosis, by radiological screening and to define criteria for young players before they turn professional to prevent the risk of serious spinal cord injuries.

Methods: Thirty one players (19 forwards and 12 backs) of the professional rugby team of Toulon were screened during the season 2000-2001. Radiological protocol: standard plain lateral view, neutral position. Measures: (a) AP diameter of the canal; AP diameter of the vertebral body; Torg ratio; static and morphological abnormalities; additional MRI or computed tomography scans when necessary; correlation with clinical findings.

Results: We found five players with stenosis at one level, four players with stenosis at two levels or more, and three players with limit value of Torg ratio. The analysis of the spine showed associated lesions with static disorders, degenerative disks, and traumatic sequelae.

Conclusion: Professional rugby has become harder. In consequence, the risk of serious injury has increased. Methods for prevention of cervical injuries include radiological screening for abnormalities that could increase the gravity of spinal cord injuries. This protocol will be used by the French Rugby Union to screen 18 year old players hoping to join the 32 French professional teams next season.

\section{EFFECTS OF ACUTE DYNAMIC EXERCISE ON} HAEMOSTASIS IN FIRST CLASS FOOTBALL REFEREES

E.E. Mackie, M. Dawson, A. McKenzie, D. Hughes, W.S. Hillis. Department of Medicine and Therapeutics, University of Glasgow, 38 Church Street, Glasgow G12 8QQ, Scotland, UK

Background: It has been shown that acute exercise stimulates coagulation, fibrinolysis, and thrombocytosis, and physical fitness may confer protection from the potential prothrombotic effects of acute exercise.

Aim: To assess the haemostatic changes with acute exercise in a group of trained subjects in the age group at risk of acute coronary events.

Methods: Twenty referees performed a ramp protocol exercise test with continual expired gas analysis. Blood sampling was performed before, immediately after, and 30 minutes after exercise for platelet count, prothrombin time (PT), activated partial thromboplastin time (APTT), and tissue plasminogen activator (†PA). Platelet activation was assessed using flow cytometry. CD62 (p-selectin) and anti-fibrinogen were used at rest and in response to ADP and adrenaline (epinephrine).

Results: The mean (SD) age of the participants was 39.2 (5.1) years. Peak $\mathrm{VO}_{2}$ achieved was $47.23(5.02) \mathrm{ml} / \mathrm{kg} / \mathrm{min}$ (table).

Conclusion: Acute dynamic exercise in trained middle aged subjects appears to be prothrombotic, with significant activation of coagulation, fibrinolysis, and platelets. In contrast with previous studies in young athletes, this study suggests that an older athletic population are not protected against the prothrombotic effects of exercise, and supports a role for regular cardiovascular screening in this population for signs of reversible ischaemia.

\section{A STUDY INTO THE AVAILABILITY OF MEDICAL FACILITIES AT SCOTTISH PREMIER DIVISION RUGBY CLUBS}

Y. Kathiravel', D.A.D. Macleod'. 'Department of A\&E Medicine, Royal Infirmary of Edinburgh, Lauriston Place, Edinburgh EH3 9YW, Scotland, UK; ${ }^{2}$ The Royal College of Surgeons of Edinburgh, Nicolson Street, Edinburgh EH8 9DW, Scotland, UK

The provision of medical facilities in adult rugby clubs has not been determined to date. The proportion of players injured has almost doubled since the advent of professionalism.' Furthermore, it has been 


\begin{tabular}{|c|c|c|c|c|c|c|}
\hline \multicolumn{7}{|l|}{ Abstract 16} \\
\hline & Before & After & $\mathrm{p}$ Value & Before & $30 \mathrm{~min}$ after & $p$ Value \\
\hline Platelets & $228.2(40.5)$ & $278.6(45.8)$ & 0.001 & $228.2(40.5)$ & $240.2(45.8)$ & 0.006 \\
\hline APTT & $32.1(3.1)$ & $29.7(3.9)$ & 0.001 & $32.1(3.1)$ & $28.4(3.3)$ & 0.001 \\
\hline PT & $12.1(0.6)$ & $11.6(0.6)$ & 0.004 & $12.1(0.6)$ & $12.0(0.7)$ & 0.3 \\
\hline tPA antigen & $0.47(0.8)$ & $6.28(4.45)$ & 0.0001 & $0.47(0.8)$ & $0.9(1.1)$ & 0.09 \\
\hline$\%$ CD62 & $0.68(0.52)$ & $1.42(1.3)$ & 0.008 & $0.68(0.52)$ & $0.78(0.58)$ & 0.6 \\
\hline$\%$ Anti-fibrinogen & $5.19(4.3)$ & $13.01(14.24)$ & 0.017 & $5.19(4.3)$ & $20.47(26.8)$ & 0.02 \\
\hline
\end{tabular}

\begin{tabular}{|c|c|c|c|c|c|c|}
\hline \multicolumn{7}{|c|}{ Abstract 20} \\
\hline & \multicolumn{2}{|l|}{ MIP } & \multicolumn{2}{|l|}{$\mathrm{Vo}_{2} \operatorname{MAX}$} & \multicolumn{2}{|l|}{$\mathrm{T}_{\text {LIM }} 75$} \\
\hline & Before & Aftert & Before & After & Before & After \\
\hline IMT & 131 (46) & $185(41)^{*}$ & $3.7(0.5)$ & $3.8(0.6)$ & $60(13)$ & $82(15)^{*}$ \\
\hline SHAM & $136(29)$ & $140(29)$ & $3.0(0.7)$ & $3.1(0.7)$ & $51(29)$ & 55 (33) \\
\hline $\mathrm{CON}$ & $127(40)$ & 128 (39) & $3.0(0.6)$ & $3.0(0.7)$ & 60 (31) & $58(30)$ \\
\hline
\end{tabular}

shown that $27 \%$ of injury episodes were treated at the side of the pitch. ${ }^{2}$ This study aims to determine the availability of medical equipment, trained personnel, and mechanisms for recording injuries in rugby clubs in Scotland.

Questionnaires were circulated to the 10 clubs in each of the three Premier Divisions during the 2001-2002 season. A response rate of $100 \%, 75 \%$, and $55 \%$ was obtained for the 1 st, 2 nd, and 3rd Division respectively. All clubs had access to a first aid room, with over $22 \%$ of clubs having access to more than on facility. Chartered physiotherapists reviewed the first aid rooms on a weekly basis.

The questionnaire included a list of approved medical equipment, but only $55 \%, 33 \%$, and $22 \%$ of clubs in the 1 st, 2 nd, and 3rd Division had a complete range of equipment at the clubhouse and on field.

Overall, $55 \%, 75 \%$, and $76 \%$ of clubs in the 1 st, 2 nd, and 3 rd Division respectively maintained a record of injured players. All clubs, except one, worked with a chartered physiotherapist, and $85 \%$ of clubs had the services of a dedicated team doctor. Some $87 \%$ of clubs had a first aid qualified team attendant.

This study shows that the level of equipment and personnel varied greatly among clubs, and great deficiencies have been identified.

1. Garraway WM et al. Br J Sports Med 2000

2. Kathiravel $Y$, et al. The management of rugby injuries. Poster presentation BASEM congress.

\section{INJURY PATTERNS AND INJURY PREVENTION STRATEGIES IN THE WINTER SPORTS POPULATION ATTENDING THE ENGLISH MEDICAL CENTRE IN VAL D'ISERE, FRANCE}

S.D.J. Reid. The English Medical Centre, Val Village, Val d'lsere, France

This study takes the unique opportunity to study a predominantly British population of skiers and snowboarders abroad.

Detailed questionnaires identifying risk factors for injury were filled in by all injured parties and completed by a doctor or student doctor during a four week period in January/February 2002.

The most common injuries among skiers were of the knee $142.5 \%$ total skiing injuries) followed by the head and neck (13.1\%), and the shoulder $(11.9 \%)$.

Snowboarders most commonly injured their wrist $(20.8 \%)$, followed by the head and neck (16.7\%) and shoulder (15.3\%).

Calf strains are poorly documented in the literature, but were found to be a significant injury $(6.3 \%$ total skiing injuries), only observed in skiers over 35 years old. Risk factors and prevention strategies were discussed for this and other common injuries where appropriate.

Men were injured more than twice as often as women, partly because of men being more aggressive slope users. The hour between 2 and 3 o'clock was found to be when slope users were most at risk of injury.
The data support the use of helmets for both skiers and snowboarders, and I would also strongly advocate the use of wrist guards by snowboarders.

\section{NINE WEEKS OF INSPIRATORY MUSCLE TRAINING AND ITS EFFECT ON CYCLING ENDURANCE}

A.D. Gething, E.M. Williams, B. Davies. Field of Health \& Exercise Science, School of Applied Sciences, University of Glamorgan, Glamorgan CF37 IDL, Wales, UK

Background: The respiratory muscles can be specifically trained for both strength and/or endurance.' The effect that this will have on exercise performance remains equivocal despite continued interest within the literature. ${ }^{2,3}$

Aim: To assess the effect of a through-volume fixed load inspiratory muscle trainer (IMT) on cycling endurance.

Method: Fifteen subjects were randomly assigned to one of three groups. Five subjects underwent IMT using the TIRE device, and five performed sham IMT with a greatly reduced resistance to flow. Training was performed three times a week for nine weeks. A further five subjects acted as controls and received no training. Lung function, maximum inspiratory pressure (MIP), $\mathrm{VO}_{2} \mathrm{MAX}$, and time to exhaustion at $75 \% \mathrm{VO}_{2} \mathrm{MAX}\left(\mathrm{T}_{4 \mathrm{M}} 75\right)$ were measured before and after the intervention. Heart rate, ventilation, and rating of perceived exertion (RPE) were also measured during the trials.

Results: IMT using the TIRE device significantly improves MIP, an indicator of inspiratory muscle strength. This, in turn corresponds to a $36 \%$ increase in $\mathrm{T}_{U M} 75$ with a decrease in exercising heart rate, ventilation, and RPE that was not observed in either the SHAM or the CON group. There was no effect on $\mathrm{VO}_{2} \mathrm{MAX}$ or lung function in any of the groups.

Conclusion: These results show that IMT is not due to the placebo effect as previously suggested. ${ }^{2}$

1. Leith DE, Bradley M. J App/ Physiol 1976

2. Sonetti DA et al. Respir Physiol 2001

3. Romer LM et al. Med Sci Sports Exerc 2002

\section{EVIDENCE OF HYPERHOMOCYSTEINAEMIA FOLLOWING LONG TERM ANABOLIC ANDROGENIC STEROID (AAS) USE}

F.M. Grace', N. Sculthorpe', M.T. Graham', J.S. Baker', T. Garvette ${ }^{2}$, D. Hullin'2, B. Davies'. 'Department of Health \& Exercise Science, University of Glamorgan, Pontypridd, Mid-Glamorgan, Wales, UK; ${ }^{2}$ Department of Pathology, Royal Glamorgan Hospital, Mid-Glamorgan, Wales, UK

Aim: To examine the effects of long term ( $>20$ years) administration of anabolic androgenic steroids (AAS) on plasma homocysteine (HCY), vitamin B12, and folate concentrations. 
Methods: Subjects $(n=40)$ were divided into four distinct groups: AAS users $(n=10)$ who were still using at time of testing (SU), a group of AAS users $(n=10)$ who had been abstinent from AAS administration for more than three months before examination $(S A)$, bodybuilding controls $(n=10)$ who did not use any pharmacological ergogenic aids $(\mathrm{BC})$, and sedentary male controls $(\mathrm{SC})(\mathrm{n}=10)$.

Results: $\mathrm{HCY}$ was significantly higher in SU compared with $\mathrm{BC}, \mathrm{SC}$ $(p<0.01)$, and $S A(p<0.05)$. Fat free mass was significantly higher in both groups of AAS users $(p<0.01)$. Daily energy intake and daily protein intake $(\%)$ were significantly higher $(p<0.05)$ in SU and SA compared with $\mathrm{BC}$ and $\mathrm{SC}$ groups, but was unlikely to be responsible for the observed HCY increases. Haematology was unremarkable between the groups except for the packed cell volume which was significantly higher $(p<0.01)$ in the SU group. A significant inverse relation was observed between sex hormone binding globulin and $\mathrm{HCY}$, particularly in the SU group $(r=-0.828 ; p<0.01)$, indicating a possible influence of the sex hormones in determining $\mathrm{HCY}$ levels.

Conclusion: With the mounting evidence linking the capacity for AAS to adversely affect a number of clotting factors, the significantly higher levels of HCY and packed cell volume observed in the SU group suggests that long term AAS users are at increased risk of developing future thromboembolytic events.

\section{EFFECTS OF LONG TERM ANABOLIC ANDROGENIC STEROID (AAS) ADMINISTRATION ON INDICES OF RESPIRATORY FUNCTION}

F.M. Grace, A.D. Gething, M. Reilly, M. Williams, B. Davies. Department of Health \& Exercise Science, University of Glamorgan, Pontypridd, Mid-Glamorgan, Wales, UK

Aim: To investigate the effects of long term anabolic androgenic steroid (AAS) administration on respiratory function.

Methods: Subject groups consisted of AAS users $(n=9)$ who were still using AAS at time of testing (SU), AAS users $(n=6)$ who had been abstinent for more than three months (SA), bodybuilding controls $(\mathrm{n}=$ 8) $(B C)$, and sedentary male controls $(n=8)(S C)$. FEV, FVC, and PEF were measured and reported as percentage of predicted values.

Results: All subjects were within normal range, and there were no differences between groups. Maximum inspiratory pressure (MIP), an index of inspiratory muscle strength, and grip strength were both significantly greater in SU $(p<0.05)$ compared with group SC, with no significant difference found in MIP or grip strength between the other groups. MIP and grip strength showed significant correlation $(r=$ $0.57 ; p<0.05)$. There were no differences in the breathing profiles of any of the groups studied.

Conclusion: The data from this study suggest that the combination of resistance training and AAS administration produces a significant increase in MIP in a cohort of long term AAS users. Although the use of AAS is associated with a variety of potentially hazardous consequences on cardiovascular and hepatic health, we were unable to show any adverse effect of long term androgen administration on respiratory function. The efficacy of adopting AAS into clinical practice would seem to be plausible should further studies using therapeutic doses of AAS produce similar outcomes to those shown in this investigation.

\section{OPTIMISED AND NON-OPTIMISED HIGH INTENSITY CYCLE ERGOMETRY AND RUNNING ABILITY IN INTERNATIONAL RUGBY UNION PLAYERS}

N. Evans', J.S. Baker'. 'School of Sport, Physical Education and Leisure, University of Wales Institute Cardiff, Cyncoed Rd, Cyncoed, Cardiff, Wales CF2 6XD, UK; ${ }^{2}$ Health and Exercise Science Research Laboratory, School of Applied Sciences, University of Glamorgan, Pontypridd, Wales CF37 IDL, UK

Aim: To analyse running values generated on a running track and performance indices using two 30 second friction loaded high intensity protocols.

Methods: International rugby union players, 10 backs 22.7 (1.9) years; $177.1(4.5) \mathrm{cm} ; 75.6(5.6) \mathrm{kg})$ and 10 forwards $(24.1(3.5)$ years; $182.2(6.8) \mathrm{cm} ; 88.6(4.8) \mathrm{kg})$, underwent two separate friction loaded protocols (non-optimised and optimised) and five sprint running tests $(30,50,100,150$, and $200 \mathrm{~m})$.

Results: Best absolute peak power outputs were generated by the forwards (1100 (137.3) and 1272 (170.5) W; $p<0.01)$ for non-optimised and optimised protocols respectively. Forwards also elicited the highest mean power (825.8 (68.8) and 848.8 (107.1) W; $p<0.01)$. Backs were significantly faster $(p<0.01)$ than forwards over all distances. Values recorded for power output during the tests were only moderately correlated with sprint running times.

Conclusion: Results of this study suggest that cycle ergometry optimisation protocols produced significantly greater $(p<0.01)$ power outputs and blood lactate concentrations when compared with standard resistive forces. The findings also indicate that high intensity cycle ergometry is only a moderate predictor of running ability in rugby union players.

\section{STRENGTH AND BODY COMPOSITION PARAMETERS SINGLE VERSUS TRIPLE SET RESISTANCE TRAINING PROGRAMMES}

R.S. Williams, G.J. Evans, J.A. Lewis, J.S. Baker, B. Davies. Health and Exercise Science Research Unit, School of Applied Sciences, University of Glamorgan, Pontypridd CF37 IDL, UK

Aim: To analyse the effects of increasing training volume from one to three sets on muscular strength and body composition in a group of recreational weight trainers.

Methods: Training was conducted three times a week using one set $(n=8)$ or three sets $(n=8)$ of six repetitions to fatigue using variable resistance training exercises. A battery of upper body exercises consisting of the bench press, biceps curl, and shoulder press were evaluated over eight weeks using the one repetition max method (1RM).

Results: All training groups significantly improved muscular strength $(p<0.05)$ with no differences observed between the one and three set group $(p>0.05)$. Positive decreases were also observed in body fat for the one set group only $(p<0.05)$.

Conclusion: One set of high intensity resistance training was as effective as three sets for increasing upper body muscle strength. The one set protocol also produced greater decreases in body fat in a group of recreational weight trainers over an eight week time frame.

\section{OBSERVATIONAL STUDY OF THE USE OF GUM SHIELDS AND FACIAL VISORS IN BRITISH PROFESSIONAL ICE HOCKEY}

\section{N.W.A. Elliott. Dundee, Scotland, UK}

Background: Facial injuries in contact sports are well documented. Ice hockey is considered to be one of the highest risk sports with high injury rates, most notably to the face and head. The rules of the International Ice Hockey Federation stipulate the use of protective equipment, including helmets and facial protection. The use of gum shields (or mouth guards) is not mandatory. Current literature as well as that dating from the early research into the protective qualities of gum shields quite clearly shows the reduction in injury to dentoalveolar tissue as well as some effect on concussion.

Aim: To determine the current usage of gum shields and additional facial protection by players in the professional British National lce Hockey League. The hope is to highlight current usage and promote safety issues at a professional level.

Methods: Teams playing in the British National League in the 2001-2002 season were observed at warm up and match time. Players not clearly seen during these periods were approached after the matches to confirm their use of a gum shield as well as to document additional facial protection. Details recorded during matches were player position, gum shield use, and type of facial protection used.

Results: A total of 160 players (age range 17-38 with most in their mid-20s) were observed over 18 weeks (18 games). Mean gum shield use during competitive games was $52 / 160(32.5 \%$ (range $11-94 \%)$ ). The team with a maxillofacial surgeon had the highest rate of gum shield use. All goalkeepers used cages for facial protection whereas only $68 / 140(48.6 \%$ (range $29-63 \%)$ ) of outfield players wore a visor or cage. Three clubs employ the services of medical personnel (two GPs and one maxillofacial surgeon), a paramedic covers one team, and the remainder have "first-aid" staff available at matches. The three teams that employ medical personnel had a gum shield usage rate of $22 / 48(45.8 \%$ (range $13-94 \%))$ and a visor usage rate of $21 / 42(50 \%(43-54 \%))$.

Conclusions: This brief study shows that the number of players wearing facial protection in such a high risk sport is low. Professional ice hockey is a growing sport in the United Kingdom. The governing body as well as those involved as health professionals should take these findings seriously and implement education programmes for the teams involved as well as promoting the use of protective equipment at youth development level. Review of the ISL league as well as the amateur leagues would no doubt reveal a similar picture. Injury 
prevention is paramount to effective sports medicine. It also raises the question about role of the employer (the club) in preventing injury to their employees (the players).

\section{THE CASE FOR ACUTE ANTERIOR CRUCIATE LIGAMENT RECONSTRUCTION}

C.T.J. Servant, N. Bradbury, M.D. Holt, M.J. Cross. Australian Institute of Musculoskeletal Research, 286 Pacific Highway, Crows Nest, NSW 2065, Australia

Background: Arthrofibrosis following anterior cruciate ligament reconstruction (ACLR) prevents the patient from regaining full knee movement

Aim: To determine whether acute reconstruction (performed within three weeks of injury) is associated with an increased risk of arthrofibrosis compared with chronic reconstruction (performed more than eight weeks after injury).
Methods: Sixty two acute and 52 chronic patellar tendon ACLRs were included in the study. A standard surgical technique and accelerated rehabilitation programme were used in all cases. All patients were assessed independently at least three months after surgery.

Results: There was no significant difference in the incidence of arthrofibrosis between acute and chronic ACLR. Flexion of less than $125^{\circ}$ or a loss of extension of more than $10^{\circ}$ occurred in eight $(12.9 \%)$ of the acute group and in nine $(17.3 \%)$ of the chronic group. All knees were clinically stable, but the mean KT1000 difference was $1.21 \mathrm{~mm}$ in the acute group and $1.89 \mathrm{~mm}$ in the chronic group $(p<0.05)$. There were also significantly more meniscal injuries $(65 \% \mathrm{v}$ $31 \%)$ and chondral lesions (31\% v18\%) in the chronic group.

Conclusions: Acute ACLR is not associated with an increased risk of arthrofibrosis. However, it is associated with increased stability and less meniscal and chondral pathology. 